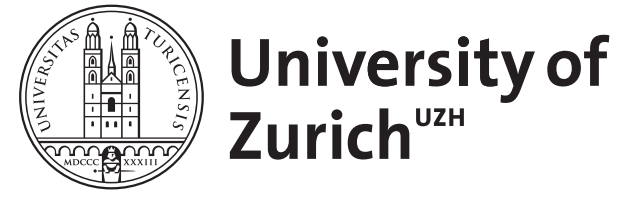

\title{
Wer was wovon lernen könnte
}

Leist, Anton \begin{abstract}
Beck Verlag, München 2012, 237 S
DOI: https://doi.org/10.1524/dzph.2012.0079

Posted at the Zurich Open Repository and Archive, University of Zurich ZORA URL: https://doi.org/10.5167/uzh-154305

Journal Article

Published Version
\end{abstract}

Abstract: Herbert Schnädelbach: Was Philosophen wissen und was man von ihnen lernen kann. C.H.

Originally published at:

Leist, Anton (2012). Wer was wovon lernen könnte. Deutsche Zeitschrift für Philosophie, 60(6):1030-1033. DOI: https://doi.org/10.1524/dzph.2012.0079 


\title{
Wer was wovon lernen könnte
}

\author{
Von ANTON LEIST (Zürich)
}

\author{
HERBERT SCHNÄDELBACH: WAS PHILOSOPHEN WISSEN UND WAS MAN VON \\ IHNEN LERNEN KANN. C. H. Beck Verlag, München 2012, 237 S.
}

In gewisser Weise handelt die Philosophie vom durchschnittlichen Leben, ihre Themen behandeln das Allgemeinste, dem sich im Leben begegnen lässt. Einige ihrer zentralen Themen, wie Geist, Sein, Erkennen, Bedeutung, sind im Alltag präsent, denn wie anders könnte ein Alltag ein menschlicher sein ohne sie? Wenn hingegen ein Phänomen, wie etwa Lautsprache, Sozialisation oder Farbwahrnehmen, nicht mehr auf derselben Allgemeinheitsstufe steht, bemächtigen sich auch bereits Wissenschaften seiner. Die Philosophie ist unumstritten die Disziplin von den ,elementaren“ - sollen wir sagen „Inhalten“, „Rahmenbedingungen“, „Gegenständen" des menschlichen Lebens? Aber, wie eigenartig, eben dadurch wird sie zugleich dem menschlichen Leben am Fernsten. Denn im Unterschied zu den ,Themen“ sind die entsprechenden „Begriffe“ im alltäglichen Leben nicht präsent. Und mehr noch: Die ,allgemeinste Wissenschaft" wird, so scheint es, im konkreten Leben nicht gebraucht, ja sie scheint mit ihm sogar unvereinbar. Eine „Alltagsphilosophie“ steht im Verdacht, keine Philosophie zu sein.

Damit wirft sich nicht nur die Frage auf, warum die Philosophie durchschnittliche Alltagsmenschen interessieren sollte, sondern auch, wie sich philosophische Erkenntnis legitimiert, wenn sie keine Verbindung mit der Alltagserfahrung hat. Die in Anführungszeichen gesetzten Wörter („Inhalte“, „Rahmenbedingungen“ etc.) werden unverständlich, wenn sie auf eine nicht-alltägliche Weise gebraucht werden sollen. Die sich damit andeutende Kluft ist an der klassischen, vorrangig an Erkennen und Geist orientierten europäischen Philosophie - beginnend mit der zweiten Hälfte des 19. Jahrhunderts - zunehmend kritisiert worden. Schopenhauer, Nietzsche, Kierkegaard, Marx, Dilthey stehen auch für eine Veralltäglichung der Philosophie, die dann im 20. Jahrhundert von den klassischen amerikanischen Pragmatisten, den deutschen philosophischen Anthropologen und von Heidegger und Sartre fortgeführt wurde. Den Nichtphilosophen die Philosophie näher zu bringen wird deshalb, sollte man meinen, am besten mit dieser Bewegung gegen die klassische Philosophie ansetzen.

Bereits das Aufscheinen des Begriffs „Wissen“ im Titel von Herbert Schnädelbachs Buch, das mindestens auch für philosophische Laien gedacht ist, deutet an, dass es diesen Weg nicht gehen will. Natürlich könnte es sich bei dem von ihm zitierten ,philosophischen Wissen“ auch um eines vom Wollen, vom Lebensernst, vom praktischen Erfolg oder vom Tod handeln. Ein Blick in das Inhaltsverzeichnis zeigt aber schnell, dass in 14 Kapiteln vorrangig der rein kognitive Mensch behandelt wird (Kap. 2-9), knapp ergänzt mit dem wertenden und handelnden (Kap. 10-12). Insgesamt finden Heidegger, Plessner und Gehlen in den begriffsgeschichtlichen Skizzen an nötiger Stelle durchaus Erwähnung. Aber Schnädelbachs ,,philosophisches Wissen“ ist im Endeffekt ein sprachphilosophisch geläutertes „Wissens-Wissen“, und nicht ein Wissen vom vielleicht interessanteren Rest im menschlichen Leben. Eigenartigerweise 
konstatiert Schnädelbach selbst wiederholt die Alltagsferne der philosophischen Begriffe (42, $58,87,100,110,128,176,192)$, zieht daraus aber weder kritische methodische Folgerungen, noch geht er auf die Ursachen dieser Kluft näher ein.

Gegeben Titel und Absicht, setzt das Buch naheliegenderweise mit dem Wissensbegriff ein, geht dann aber unvermittelt von der spezifischen Frage des „philosophischen Wissens“ über zur Frage vom Wissen im Allgemeinen. Wird zuerst noch Wittgensteins Diktum von Philosophie als Tätigkeit zitiert (28), entfalten die nächsten Kapitel die zentralen Themen der modernen Philosophie: Wissen, Wahrheit, Sinn, Bedeutung, Denken, Selbstbewusstsein. Die häufig von der Antike bis zur Neuzeit durchlaufende Schilderung dieser Begriffe lässt die metaphilosophische Thematik meist in der Schwebe. Erst im letzten, mit der analytisch/ synthetisch-Unterscheidung befassten Kapitel legt Schnädelbach seine Karten auf den Tisch. Nun bekennt er sich zu einer „erläuternden“ begriffsanalytischen Philosophie, für die er nicht nur in Quines berühmtem Artikel, sondern auch bereits in Kants Selbstverständnis der Kritik der reinen Vernunft einen Beleg findet (222). Das „Wissen der Philosophen“, so muss man Schnädelbach zusammenfassen, ist das einer Begriffserläuterung prinzipiell im Stil Kants, wenn auch ohne apriorischen Anspruch, und soweit passend, wie im Fall des Gestaltsehens (220), durchaus informiert durch Wissenschaft.

Diesem Selbstverständnis gehen voran mehrere Illustrationen der neueren Erfolgsgeschichte der Philosophie, die Schnädelbach in der Überwindung des mentalistischen Paradigmas durch den „linguistic turn“ sieht. Die informativsten Kapitel schildern die Schwierigkeiten, in die das Nachdenken über das Denken gerät, wenn es die fundierende Funktion der Sprache übersieht. Die Darstellung setzt ein mit der Entdeckung der Rolle der Urteile. Erstens wird gezeigt, inwiefern es so etwas wie eine „Seinswahrheit“, die auf unmittelbarer Gewissheit beruhte, im Unterschied zur Urteilswahrheit nicht geben kann (Kap. 2, bes. 33 ff.). Zweitens wird daran erinnert, wie sich unterschwellig bereits bei Kant die Wissensfrage hin zur Verstehensfrage verschiebt, die sprachliche Bedeutung aber nicht aus der Schicht mentaler oder realistisch vorgestellter Phänomene heraus, sondern nur intern aus dem sprachlichen Gebrauch erklärt werden kann (Kap. 3, bes. 45, 51 f.). Auch die „Referenz“ auf Dinge setzt, um erfolgreich zu sein, bereits Bedeutung voraus.

Drittens werden die Schwierigkeiten geschildert, in die Aristoteles', Lockes und Kants Synthesistheorie beim Erklären der Urteile gerät, und wird gezeigt, wie erhellend im Kontrast die semantische Funktionstheorie an diesem Punkt verfährt (Kap. 4, bes. 62 ff.). Viertens wird daran erinnert, inwiefern das Verhältnis von Denken und Sprechen in der mentalistischen Tradition als instrumentell gedacht werden musste und wie langsam sich die Einsicht in die Identität von Sprache und Denken über Herder, Humboldt, Cassirer und Wittgenstein durchzusetzen hatte, ankämpfend gegen gewichtige Rückfälle bei Husserl und Heidegger (Kap. 5).

Vielleicht sollte man dieses letzte (5.) Kapitel zusammenfassend verstehen. Denn die jetzt folgenden Kapitel gelten eher der mentalistischen Psychologie selbst. So wird gezeigt, wie schwer sich die Rede vom „Ich“ auf mentale Vorstellungen zurückführen lässt, im Unterschied zum öffentlichen Vorführen des Gebrauchs von Personalpronomina (Kap. 6). Dann wird die Rede von Sätzen und Tatsachen als insbesondere der im Deutschen Idealismus favorisierten Subjekt-Objekt-Metapher überlegen geschildert (Kap. 7). Schließlich soll die sprachanalytische Methode am Erklären von Selbstbewusstsein vorteilhaft erscheinen (Kap. 8). Dieses letzte Kapitel bleibt aber eigenartig unentschieden.

Dabei versäumt Schnädelbach zunächst die Gelegenheit, auf die fragwürdige Erfindung Kants hinzuweisen, wenn er mit der Unterscheidung zwischen einer ,transzendentalen Apperzeption“ im Kontrast zur empirisch-psychologischen Reflexion ein Regressproblem beheben will (114 ff.). Schnädelbach scheint diese Antwort ebenfalls nicht völlig befriedigend 
zu finden, denn er schildert danach Ernst Tugendhats wittgensteinianische These von der „kognitiven Asymmetrie und der veritativen Symmetrie“ in der Anwendung psychologischer Prädikate, sowie zugleich Manfred Franks Zirkeleinwand gegen diese These, ohne sich für die eine oder andere Position erwärmen zu können. Wie öfters in dem Buch, endet diese Geschichte mit dem unbefriedigenden Hinweis auf anhaltende Kontroversen (125). Nach Schnädelbachs Fortschrittsthese müsste aber Tugendhats Angebot gegenüber den Versionen Kants und Franks den Sieg davontragen, und es bleibt unklar, warum das nicht der Fall ist und was das für die Sprachanalyse bedeutet.

Schnädelbachs Unentschiedenheit ist allerdings auch Ausdruck davon, dass er den ,linguistic turn" im Wesentlichen nur als eine Wende in der Methode versteht, und nicht als eine Veränderung des menschlichen Selbstverständnisses. Obwohl auch dieser Punkt unklar bleibt, ist doch vielleicht die Rede vom philosophischen als einem „,methodologischen Wissen“ (41) nicht zufällig. In Schnädelbachs Darstellung kann man den Eindruck gewinnen, dass - etwas salopp gesagt - Kants Philosophie, im Gegensatz zu dessen Nachfolgern im 19. Jahrhundert, im Grunde schon in Ordnung ist, wird sie nur entschiedener als von Kant selbst sprachanalytisch artikuliert. Was das heißen soll, wird aber in den nun noch folgenden, auf den ersten Blick praktischen Themen gewidmeten Kapiteln vollends unklar.

Diese Kapitel sind nämlich zunehmend von der völligen Offenheit der entsprechenden Probleme geprägt. Die Warnung vor dem naturalistischen Fehlschluss (Kap. 10) scheint Schnädelbach wichtig zu sein, weil er generell naturalistische Ansätze in der Philosophie für problematisch hält; argumentativ begnügt er sich gegen den Naturalismus dabei meist mit dem Vorwurf der Trivialisierung (153). Selbst Hume trivialisierend, schlägt er sich wiederum auf die Seite Kants (155), ohne allerdings dessen Vernunfttheorie zu bekräftigen (156). (Trivialisiert wird Hume, weil dessen ,künstliche Tugenden“ unterschlagen werden.) Da auch Moores Intuitionismus ausgeschlossen wird, bleibt offen, mit welchen „Prämissen“ (153) praktisch argumentiert werden soll. Der kurze Überblick zu den ethischen Positionen und auch das Kapitel über Vernunft (Kap. 13) bieten dazu keinerlei Hinweis.

Die trivialisierte Rolle der Emotionen, die Sprödigkeit gegenüber Schopenhauers Versuch, den Willen zu verleiblichen (118 f.), das Thematisieren von Handlungen und Vernunft als nur theoretischen Begriffen, von Willensfreiheit im Konflikt mit dem Determinismus (189), alle diese Stücke unterstreichen, dass Schnädelbach die hier dargestellte Philosophie theoretisch versteht. In Verbindung mit dem ansonsten uneingeschränkten Anspruch des, ,philosophischen Wissens“ (,was Philosophen wissen“) sagt das nun auch, dass er es entsprechend verkürzt. Schnädelbach zitiert an einer Stelle zustimmend Rortys Diagnose, wonach die analytische Philosophie die Erkenntnistheorie fortsetze (27), versäumt es aber, diese von Rorty kritisch gemeinte Beobachtung in seiner Darstellung anzuwenden. Rorty war natürlich nicht der Meinung, dass die Sprachanalytiker helfen sollten, die klassischen Probleme ,besser" zu bearbeiten, sondern vielmehr, dass sie sich erneut in dasselbe Denkgefängnis begeben haben, das die anthropologischen Philosophen des 19. Jahrhunderts und die amerikanischen Pragmatisten teilweise bereits überwunden hatten. Eine so radikale Diagnose dürfte Schnädelbach dem Irrationalismus (200) zurechnen, ohne dass in diesem Buch klar wird, mit welcher Berechtigung.

Gegen diese Kritik lässt sich einwenden, dass der Autor von vornherein klarstellt, dass seine Absicht, einen innerdisziplinären Konsens als „Wissen“ wiederzugeben, auf die theoretische Philosophie beschränkt bleibt (14). Ist eine solche Schwerpunktsetzung in einem überwiegend historisch-deskriptiv vorgehenden Buch nicht völlig berechtigt? Sicher, würde das Ergebnis tatsächlich einen Konsens wiedergeben. Im engeren Sinn der von Schnädelbach diskutierten Teilstücke, etwa der Untauglichkeit des Subjekt-Objekt-Modells, mag ein 
Konsens auch tatsächlich herrschen. Aber die weitgehende inhaltliche Unverbindlichkeit des „philosophischen Wissens“, die sich bei der Lektüre des Buchs zunehmend enthüllt, deutet aus sich heraus bereits an, dass hinter ihr kein Konsens stehen kann. Die sprachanalytische Erfolgsgeschichte - die im engeren Sinn nicht bestritten werden muss - gerät im Buch selbst in Zweifel, wenn die praktischen Begriffe „Werte“, „Normen“, „Handlungen“ angegangen werden.

Tatsächlich führt die bloße Begriffsanalyse bei interessanteren alltäglichen Themen wie Moral, gutes Leben, Angst, Tod, Schuld, Freiheit, Politik entweder zum Quietismus oder zur Selbstaufgabe als voraussetzungsloser Methode. Der wittgensteinsche Quietismus zeigt sich in Schnädelbachs Geschichte spätestens anhand von Tugendhats Asymmetriethese der psychologischen Prädikate (123). Diese Asymmetrie wird nur beschrieben, nicht erklärt. Die Begriffsanalyse beschreibt - etwas, was handelnde und leidende Menschen nicht zufriedenstellen kann. Sie wollen nicht nur verstehen, sondern Motive und Ziele entwickeln. Auch mögen zwar Berufsphilosophen von den Irrtümern des mentalistischen Paradigmas ,,verhext“ sein, nicht aber philosophische Anfänger, sodass ihnen gegenüber die kritische Pointe der Sprachtherapie verpufft.

Philosophisch ungebildeten Lesern dieses Buchs müsste erklärt werden, warum ihnen der interne Selbstklärungsprozess der Philosophie so wichtig sein soll. Das hier aufscheinende Problem sollten viele der heute ,analytisch“ arbeitenden Philosophen mit Schnädelbach ernst nehmen. Denn ähnlich wie Schädelbach akzeptieren sie zwar den anti-metaphysischen Fortschritt der Sprachanalyse, aber ziehen aus ihm keine weiteren Folgen als die, ihre Begriffsanalysen mit einer methodischen Fußnote zu versehen, wohingegen zu sehen wäre, mit welchen Wertverschiebungen das Gesamtmanöver verbunden ist. Ähnlich lässt Schnädelbachs im Ton immer leicht tadelnder Hinweis, dass aus der philosophischen Begrifflichkeit alle alltäglichen Bezüge getilgt sind, ein Desiderat erkennen, nicht aber, wie ihm zu begegnen ist.

Für einen philosophisch laienhaften Leser dieses Buchs sollte sich deshalb die Frage stellen, warum er nicht besser Japanisch lernen oder die Partien Bobby Fischers nachspielen sollte, als die Selbstkorrekturen einer vom Alltag losgelösten Philosophie zu studieren. Und tatsächlich gilt ungeachtet von Schnädelbachs im Großen und Ganzen sehr informativem Bericht, dass kaum ein heutiger Philosoph die allgemeine Relevanz der Philosophie vermitteln kann, ohne dazu entweder menschliche Wesensprämissen zu unterstellen oder auf Themen (etwa der Ethik) auszuweichen, für die Philosophen kein Erklärungsmonopol mehr haben. Alle haben heute erkannt: Das mentalistische Paradigma in der Philosophie hat keine Beziehungen zum durchschnittlichen Leben, sondern war eine lebensferne Verirrung. Damit ist man aber noch lange nicht auf dessen anderer Seite angekommen, nämlich einer relevanten Philosophie, die in angemessener Nähe und Ferne zum Alltag etwas zu sagen hat. Leider suggerieren Titel und manche Passagen in Schnädelbachs Buch, dass die Alltagsmenschen etwas von der heutigen Philosophie lernen könnten. Aber lernen können sie bestenfalls ihre heutige Problematik und Schwäche, ohne dass sie ihnen auch nur ansatzweise erklärt würde. Im Gegenteil ist das Buch um so etwas wie einen „soliden Bestand“ bemüht. Wieviel besser wäre es, es würde zeigen, wo sich Leben und Philosophie berühren. 